\title{
HIV-associated Tuberculosis: Clinical Case
}

\author{
S. S. Sleptsova ${ }^{1 *}$, N. A. Gulyaeva ${ }^{1}$, P. S. Dyachkovskaya ${ }^{1}$, V. K. Semenova ${ }^{1}$, \\ M. V. Egorova ${ }^{1}$, L. I. Petrova ${ }^{1}$, G. A. Krichko ${ }^{1}$, M. N. Andreev ${ }^{1}$, \\ N. N. Sofronova ${ }^{2}$, M. K. Vinokurova ${ }^{3}$, O. E. Dogorova ${ }^{3}$ \\ ${ }^{\prime}$ M. K. Ammosov North-Eastern Federal University, Yakutsk, the Sakha Republic, Russia \\ ${ }^{2} P K Z$ SPID I IZ, Perm, Russia \\ ${ }^{3} Y a k u t$ NPZ "Ftiziatriia", Yakutsk, the Sakha Republic, Russia
}

\begin{abstract}
This case report presents a 35-year-old female who presented with HIV-associated multidrug-resistant tuberculosis. Resistant tuberculosis in HIV-infected patients with immunodeficiency is extremely difficult for effective treatment. Nevertheless, our clinical case showed that the cure of tuberculosis is possible in the late stages of HIV infection with the correct choice of treatment tactics, taking into account the resistance of MBT with the subsequent use of antiretroviral therapy. The progression of HIV infection, against the background of the effective antituberculous therapy, was associated with the patient's refusal to treat the underlying disease. (International Journal of Biomedicine. 2017;7(3):257-259.)
\end{abstract}

Key Words: human immunodeficiency virus • multidrug-resistant tuberculosis • antiretroviral therapy • antituberculous therapy

\section{Abbreviations}

AIDS, acquired immune deficiency syndrome; ARVT, antiretroviral therapy; CHC, chronic hepatitis C; HIV, human immunodeficiency virus; MBT, Mycobacterium tuberculosis; MDR-TB, multidrug-resistant tuberculosis; RS(Y), the Republic of Sakha (Yakutia); TB, tuberculosis.

\section{Introduction}

According to UNAIDS, there were approximately 36.7 million people worldwide living with HIV/AIDS at the end of 2015. An estimated 2.1 million individuals worldwide became newly infected with HIV in 2015.(1) Since the beginning of the epidemic, more than 70 million people have been infected with the HIV virus and about 35 million people have died of HIV. ${ }^{(2)}$

However, between 2000 and 2015, the number of new HIV infections in the world decreased by $35 \%$ and the AIDSrelated death rate decreased by $28 \%$, which saved the lives of 8 million people. On the contrary, in the Russian Federation, the number of HIV-infected people is increasing annually. ${ }^{(3)}$ As of December 31, 2016, according to official data, 103,438 new HIV infections among the citizens of Russia were

*Corresponding author: Snezhana S. Sleptsova, PhD, ScD. M. K. Ammosov North-Eastern Federal University. Yakutsk, the Sakha Republic, Russia.E-mail: sssleptsova@yandex.ru reported, which is $5.3 \%$ more than in 2015 . The total number of reported cases of HIV infection among citizens of Russia has reached more than 1.1 million people, of whom 243,836 (21.8\%) died from various causes. According to the data of the Yakut Republican Center for AIDS Prevention and Control, 2,022 HIV-infected persons have been registered since 1996 in RS(Y), and for the 6 months of 2017, 97 new cases of HIV infection were detected.

Along with HIV infection, Russia is one of 22 countries with a high burden of TB (1990-2014). In 2014, according to WHO, the prevalence rate of TB in Russia was 109[49-192] per 100,000 population, and HIV prevalence in incident TB cases was 4.6[3.8-5.3]\%. Worldwide, 9.6 million people are estimated to have fallen ill with TB in 2014. Globally, $12 \%$ of the 9.6 million new TB cases in 2014 were HIV-positive. TB now ranks alongside HIV as a leading cause of death worldwide. In 2014, TB killed 1.5 million people (1.1 million HIV-negative and 0.4 million HIV-positive). ${ }^{(4)}$

In RS(Y), 827 persons with HIV infection are currently registered, and 180 of them receive preventive therapy against 
tuberculosis. In 2015, 42 patients with HIV in combination with TB were registered.

Globally, people living with HIV are 26 times more likely to develop TB disease than those who are HIV-negative. ${ }^{(5)}$ The interdependence of the two epidemic processes is multifactorial in nature, based on the disturbances in the immune response. (6) TB in HIV-infected patients usually tends to progress and disseminate. ${ }^{(7)} \mathrm{TB}$ can occur early in the course of HIV infection and throughout all stages of the disease. Although TB can be a relatively early manifestation of HIV infection, it is important to note that the risk of developing TB, and of disseminated infection, increases as the CD4 cell count decreases. Even with effective immune reconstitution with ART, the risk of TB generally remains elevated in HIV-infected patients above the background risk of the general population, even at high CD4 cell counts. ${ }^{(8-10)}$

The presentation of TB is affected by the extent of HIVrelated immunosuppression. In patients with CD4 counts of $>350$ cells $/ \mu \mathrm{L}$, the clinical and radiographic presentation is similar to that of patients without HIV infection. However, as immunosuppression advances, the radiographic presentation becomes less typical and extrapulmonary and disseminated disease becomes more common. However, in patients with primarily extrapulmonary involvement or disseminated disease, the CD4 cell count may be much lower.

Similarly, TB may negatively impact the natural history of HIV infection. Several studies have indicated that TB coinfection increases the risk of HIV progression and death, particularly in persons with untreated HIV disease. ${ }^{(11,12)}$ The effect of TB on HIV disease progression is hypothesized to be attributable to increased immune activation ${ }^{(13)}$ and increased expression of the CCR5 and CXCR4 co-receptors on CD4 cells. ${ }^{(14)}$ In addition, the HIV and tuberculosis combination is related closely to resistance of MBT to anti-tuberculosis drugs. The combination of HIV infection and multidrugresistant tuberculosis (MDR) is characterized by an extremely unfavorable course. ${ }^{(3,15)}$

In medical practice, priority is the early therapy of tuberculosis with subsequent antiretroviral therapy. WHO recommendations on the interventions needed to prevent TB in HIV-positive people and to reduce the impact of HIV among TB patients were first issued in 2004, and are collectively known as collaborative TB/HIV activities. ${ }^{(4)}$

\section{Case report}

A 35-year-old woman with the antibodies to HIV infection detected in June 2011 was registered in the Yakutsk AIDS-Center in 2012. The infection was associated with intravenous drug use for 5 years. CHC was detected in 2012, but the antiviral treatment for $\mathrm{CHC}$ was not received.

In 2015, she was hospitalized in the infectious department of YaGKB with a diagnosis of active cytomegalovirus infection on the background of HIV-infection stage 4B. She had a fever of $39^{\circ} \mathrm{C}$, abdominal pain, a loose stool 1-2 times a day, and general pronounced weakness. At the examination, CD 4 counts of $>289$ cells $/ \mu \mathrm{L}, \mathrm{RNA}-\mathrm{HIV}-627,000$ copies $/ \mathrm{ml}$. The patient refused ARVT.
At a repeated examination on August 24, 2016, the diagnosis was as follows: «HIV infection, stage 4B, the progression phase in the absence of ARVT; AIDS. Cytomegalovirus infection; and the Epstein-Barr infection. CHC».

Complaints: a fever of $39{ }^{\circ} \mathrm{C}$, abdominal pain, loose stools 1-2 times a day, marked weakness, cough, and weight loss. Health deteriorated during the last month, when these symptoms appeared.

Objective data: General condition was of moderate severity. Consciousness was preserved. The position in bed was passive. Skin was clean, dry, and pale. Local cyanosis and rash were absent. Lips were covered with crusts. Sclera of the eyes was a normal color. Subcutaneous fatty tissue was poorly developed. Edemas and bedsores were not present. Submandibular, cervical and inguinal lymph nodes were enlarged to $0.5 \mathrm{~cm}$, dense, and painless. Throat was slightly hyperemic, tonsils not enlarged. The frequency of respiratory movements was 18 per minute. During auscultation, wheezing was not present. Heart sounds were rhythmical, muffled. Abdomen was soft, sensitive along the intestine, peristalsis preserved. The liver edge had a soft-elastic consistency. Spleen was not enlarged. The stool was regular and liquid; color was brown without pathological impurities. Urination was free, painless. Diuresis was adequate. Neurological status: meningeal symptoms were absent.

Laboratory test data: The total blood test (08.25.16): leukocytes $-3.36 \times 10^{9} / 1$, erythrocytes $-5.5 \times 10^{12} / 1$, hemoglobin - $137 \mathrm{~g} / \mathrm{l}$, hematocrit $-39.3 \%$, lymphocytes - $8 \%$, monocytes $-8.3 \%$, neutrophils $-82.5 \%$, eosinophils - $1.2 \%$, basophils $0 \%$, platelets $-89 \times 10^{9} / \mathrm{L}, \mathrm{ESR}-32 \mathrm{~mm} / \mathrm{h}$.

Biochemical blood test (08.25.16): albumin - $34.5 \mathrm{~g} / \mathrm{l}$, ALT - $10 \mathrm{U} / \mathrm{L}$, AST $-21.9 \mathrm{U} / \mathrm{L}$, total bilirubin - $4 \mu \mathrm{mol} / \mathrm{l}$, cholesterol - $2.01 \mathrm{mmol} / \mathrm{l}$, creatinine - $79 \mu \mathrm{mol} / \mathrm{l}$, glucose $4.78 \mathrm{mmol} / \mathrm{l}$, total protein $-80.9 \mathrm{~g} / 1$, urea $-4 \mathrm{mmol} / \mathrm{l}$.

Cytomegalovirus (CMV) and Epstein Barr Virus (EBV) were detected in PCR (07.21.16). In PCR (07.21.16), the number of HIV RNA copies was 2, 400, 000 copies $/ \mathrm{ml}$.

Immunological examination of blood (07.19.16): leukocytes - 6.2×10\%/1, lymphocytes 10\% (19-37), CD3 - 41\% (60-80), CD4 - 9\% (55 cells/ml), SD8 - 30\% (16-39).

EIA (08.25.2016): HbsAg - negative, anti-HBcorIgM negative, anti-HbsAg - negative, $\mathrm{HBeAg}$ - negative, anti-HDV - negative, anti-HCV - positive, core (+), NS (-), anti-HCV IgM - negative.

CT chest (08.25.16): the disseminated lung process.

Ultrasound of the abdominal cavity (08.25.16): moderate lymphadenopathy, diffusive changes in the liver.

Blood for sterility and blood culture $(08.26 .16)$ was negative.

Smear from fauces for candidias (08.26.16): Candida albicans $>1 \times 10^{6} / \mathrm{ml}$, sensitive to nystatinum.

PCR (08.26.16): CMV DNA was not detected, HSV RNA was not detected.

Sputum smear on MBT by the bactec method (08.29.16): MBTs were detected and resistant to streptomycin, isoniazid, rifampicin, kanamycin, and capreomycin. All sputum cultures were negative from September to December 2016. Sputum on 
MBT by the real-time PCR method (08.26.16): MBT resistant to rifampicin.

Based on clinical and anamnestic results of the examination, the following diagnosis was constructed: "HIV infection, 4B stage, the progression phase in the absence of ARVT. AIDS. Disseminated pulmonary TB in the phase of infiltration. MBT(+). MDR-TB: streptomycin, isoniazid, rifampicin, kanamycin, capreomycin. Candidiasis of the oral mucosa. Replicative chronic viral hepatitis C."

On August 31, 2016, the patient was transferred with this diagnosis for treatment to the Department of Multiple Drug Resistance of the Yakut NPZ "Ftiziatriia", where she was treated for 75 days and received treatment according to the IV standard regime (pyrazinamide, ethambutol, amikacin, levofloxacin, cycloserine, para-aminosalicylic acid). The patient refused ARBT treatment. Due to side effects in the form of pain throughout the body, and weakness, aminosalicylic acid was replaced by prothionamide; ethambutol was canceled due to the contra-indication of the ophthalmologist. Patient was hospitalized again in March 2017 and underwent treatment according to the IV regime (levofloxacin, pyrazinamide, cycloserine, protionamide). She received only 12 doses. On a review of the chest X-ray (13.03.2017): The dynamics were positive; we detected a compaction of foci in S6 of both lungs. At the insistence of the patient, in connection with the move to another place of residence, she was discharged on 04.12.17, while the level of CD4 decreased up to 5.2 cells, the viral load was 3,350,000 copies/ml. Patient again refused ARBT treatment.

\section{Conclusion}

Resistant tuberculosis in HIV-infected patients with immunodeficiency is extremely difficult for effective treatment. Patients' adherence to treatment also plays a significant role in recovery. Detection and treatment of tuberculosis in HIVinfected patients at an early stage of HIV infection is most effective. Nevertheless, our clinical case showed that the cure of tuberculosis is also possible in the late stages of HIV infection with the correct choice of treatment tactics, taking into account the resistance of MBT with the subsequent use of ARVT.

Thus, patients with HIV infection require professional psychological support with training and conversation. In our clinical case, the progression of HIV infection, against the background of the effective antituberculous therapy, is associated with the patient's refusal to treat the underlying disease, which significantly worsens the prognosis.

\section{Competing interests}

The authors declare that they have no competing interests.

\section{References}

1. The Global HIV/AIDS Epidemic. Available from: https:// www.hiv.gov/hiv-basics/overview/data-and-trends/globalstatistics

2. Global Health Observatory (GHO) data. HIV/AIDS. Available from: http://www.who.int/gho/hiv/en/

3. Vasilyeva IA, Pokrovsky VV, Aksenova VAB Mariandyshev AO, Ergeshov AE, Chernousova LN, Zimina $\mathrm{VN}$, et al. Federal clinical guidelines for the diagnosis and treatment of tuberculosis in patients with HIV infection. M.Tver: "Triada"; 2014. [in Russian].

4. Global Tuberculosis report 2015 WHO. Available from: http://www.who.int/tb/publications/global_report/gtbr15 main text.pdf

5. TB/HIV facts 2015 Available from: http://www.who.int/ hiv/topics/tb/tbhiv_facts_2015/en/

6. Kornilova ZKh, Lukonina IV, Alekseeva LP. [Tuberculosis concurrent with HIV infection]. Tuberk Biolezni Legkih. 2010;(3):3-9. [Article in Russian].

7. Eramova I, Matic S, Mun M. Tuberculosis and HIV infection: management tactics of patients with co-infection. Clinical protocol for the WHO European Region. 2006: 8-23.

8. Moore D, Liechty C, Ekwaru P, Were W, Mwima G, Solberg $\mathrm{P}$, et al. Prevalence, incidence and mortality associated with tuberculosis in HIV-infected patients initiating antiretroviral therapy in rural Uganda. AIDS. 2007;21(6):713-9.

9. Van Rie A, Westreich D, Sanne I. Tuberculosis in patients receiving antiretroviral treatment: incidence, risk factors, and prevention strategies. J Acquir Immune Defic Syndr. 2011;56(4):349-55. doi: 10.1097/QAI.0b013e3181f9fb39.

10. Gupta A, Wood R, Kaplan R, Bekker LG, Lawn SD. Tuberculosis incidence rates during 8 years of follow-up of an antiretroviral treatment cohort in South Africa: comparison with rates in the community. PLoS One. 2012;7(3):e34156. doi: 10.1371/journal.pone.0034156.

11. Badri M1, Ehrlich R, Wood R, Pulerwitz T, Maartens G. Association between tuberculosis and HIV disease progression in a high tuberculosis prevalence area. Int J Tuberc Lung Dis. 2001;5(3):225-32

12. López-Gatell H, Cole SR, Hessol NA, French AL, Greenblatt RM, Landesman S, et al. Effect of tuberculosis on the survival of women infected with human immunodeficiency virus. Am J Epidemiol. 2007;165(10):1134-42.

13. Vanham G, Edmonds K, Qing L, Hom D, Toossi Z, Jones B, et al. Generalized immune activation in pulmonary tuberculosis: co-activation with HIV infection. Clin Exp Immunol. 1996;103(1):30-4.

14. Wolday D, Tegbaru B, Kassu A, Messele T, Coutinho $\mathrm{R}$, van Baarle D, Miedema F. Expression of chemokine receptors CCR5 and CXCR4 on CD4+ T cells and plasma chemokine levels during treatment of active tuberculosis in HIV-1-coinfected patients. J Acquir Immune Defic Syndr. 2005;39(3):265-71.

15. Bondarenko VN. National clinical guidelines for the diagnosis and treatment of HIV infection in adults. M.; 2014. [in Russian]. 